\title{
Optimal Transmission Scheduling for a Hybrid of Full- and Half-Duplex Relaying
}

\section{AUTHOR(S):}

Yamamoto, Koji; Haneda, Katsuyuki; Murata, Hidekazu; Yoshida, Susumu

\section{CITATION:}

Yamamoto, Koji ... [et al]. Optimal Transmission Scheduling for a Hybrid of Full- and HalfDuplex Relaying. IEEE Communications Letters 2011, 15(3): 305-307

\section{ISSUE DATE:}

2011-03

URL:

http://hdl.handle.net/2433/139538

\section{RIGHT:}

(c) 2011 IEEE. Personal use of this material is permitted. Permission from IEEE must be obtained for all other uses, in any current or future media, including reprinting/republishing this material for advertising or promotional purposes, creating new collective works, for resale or redistribution to servers or lists, or reuse of any copyrighted component of this work in other works.; この論文は出版社版でありません。引用の際には出版社版をご確認ご利用ください。; This is not the published version. Please cite only the published version. 


\title{
Optimal Transmission Scheduling for a Hybrid of Full- and Half-Duplex Relaying
}

\author{
Koji Yamamoto, Member, IEEE, Katsuyuki Haneda, Member, IEEE, Hidekazu Murata, Member, IEEE, \\ and Susumu Yoshida, Member, IEEE
}

\begin{abstract}
Full-duplex relaying (FDR), i.e., simultaneous transmission and reception using the same frequency channel at a radio relay, can be used to achieve a spectral efficiency higher than that in the case of half-duplex relaying (HDR) if loopback interference is well-managed. To achieve spectral efficiency that is higher than that achieved when using FDR and HDR separately, an optimal transmission-scheduling scheme for an FDR-HDR hybrid is proposed. The scheme is formulated as an optimization problem. The conditions required to achieve the maximum spectral efficiency are determined analytically. Numerical results confirm that the proposed scheme is superior to FDR and HDR.
\end{abstract}

Index Terms-Full-duplex relaying, half-duplex relaying, transmission scheduling, linear programming.

\section{INTRODUCTION}

$\mathbf{T}$ HERE are two duplex modes for relaying: half-duplex mode and full-duplex mode. In half-duplex relaying (HDR), orthogonal time slots or orthogonal frequencies are used, and thus, there is no co-channel interference. As a result, the signal-to-interference-plus-noise ratio (SINR) in HDR is higher than that in full-duplex relaying (FDR), which suffers from co-channel interference. On the other hand, FDR has gained importance in recent years because of its high capacity potential. In the FDR mode, a relay receives and transmits data simultaneously on the same frequency channel. Thus, co-channel interference, which is also known as loop-back interference, is observed at the relay station. However, if the isolation between the receiving and transmitting antennas at the relay is infinite, the spectral efficiency of two-hop relaying in the FDR mode will be twice that in the HDR mode. This difference is because two orthogonal channels need not be used for two-hop relaying in the FDR mode. Thus, the main challenge involved in realizing FDR is to achieve high isolation between the receiving and transmitting antennas. To this end, loop-back interference canceller [?] and a compact antenna that helps realize high isolation [?] have been developed.

When there is a difference between the data rates of source-to-relay and relay-to-destination links, the end-to-end

Manuscript received October 12, 2010. The associate editor coordinating the review of this letter and approving it for publication was Y.-D. Lin.

K. Yamamoto, H. Murata, and S. Yoshida are with Graduate School of Informatics, Kyoto University, Kyoto, 606-8501 Japan (e-mail: kyamamot@i.kyoto-u.ac.jp).

K. Haneda is with Aalto University, Finland.

The present study was supported in part by a Grant-in-Aid for Scientific Research (A) (no. 20246067) from the Japan Society for the Promotion of Science (JSPS). The authors would like to thank Mr. Takashi Shimizu and Mr Takatoshi Kihara for their comments. K. Haneda would like to acknowledge the financial support of the post-doctoral and Finnish-Japanese Cooperation projects of the Academy of Finland, Helsinki, Finland.

Digital Object Identifier $* * *$

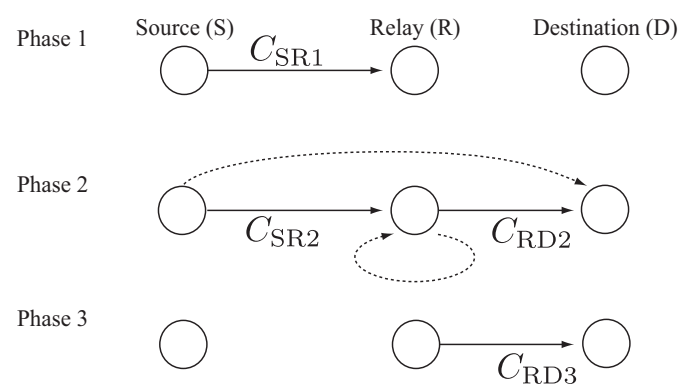

Fig. 1. Average data rate of each phase in the proposed scheme.

throughput of FDR is limited by the data rate of the weaker link, irrespective of the data rate of the stronger link. In contrast, the end-to-end throughput of HDR is affected by the data rate of the stronger link. This is a result of transmission scheduling of source-to-relay and relay-to-destination links. The optimal solution for the transmission scheduling problems encountered in HDR-based multi-hop transmission and multihop radio networks have been discussed in [?], [?], [?].

With the aim of improving the end-to-end throughput of two-hop relaying in the FDR mode, we propose a technique that uses a combination of FDR orthogonal reception and orthogonal transmission at the relay. This transmissionscheduling scheme is a hybrid of FDR and HDR. There are many papers in which the comparison of FDR and HDR is discussed [?], [?], [?]; however, there are very few papers on the combination of these two modes. Adaptive utilization of FDR and HDR has been proposed in [?], but this adaptive utilization is just a scheme that utilizes switching between FDR and HDR. However, in our hybrid scheme, an additional degree of freedom is provided to the existing switching scheme, and a high spectral efficiency is expected.

The rest of this letter is organized as follows. In Section II, the scheduling problem is formulated as a linear programming problem, and its optimal solution is devised by assuming an isolated two-hop transmission. In Section III, the optimal endto-end throughput is evaluated numerically, and it is shown that the proposed scheme is superior to both FDR and HDR. Section IV concludes the letter.

\section{Formulation AND SOLUTION OF Optimal TRANSMISSION SCHEDULING}

We consider a set comprising a source, a relay, and a destination. We assume a time duration for which the data rate is constant for each transmission between the source, relay, and destination. This assumption is reasonable in some cases, for example, when fast fading is averaged out and only the average data rate is taken into account. 
We propose a technique that is a hybrid between FDR and HDR. We also propose to optimize the end-to-end throughput by controlling the duration of each transmission. In this scheme, there are three orthogonal phases, as shown in Fig. ??. The first and third phases correspond to HDR, where the source and relay transmit using different orthogonal phases. The second phase is FDR, where the relay receives simultaneously packets from the source and transmits them to the destination. We propose to maximize the end-to-end throughput by scheduling the duration of each phase. Note that, the total amount of data transmitted in phase 1 must be equal to that in phase 3 to maximize the end-to-end throughput in general HDR, but it need not be satisfied in the proposed scheme.

Let $C_{\mathrm{XY} i}(>0)$ be the average data rate from transmitter $\mathrm{X}$ to receiver $\mathrm{Y}$ in phase $i \in\{1,2,3\}$. Since the relay and destination suffer from co-channel interference in phase 2 , the SINR for phase 2 will be lesser than that for the other phases; therefore, the average data rate in phase 2 is assumed to be lesser than that in the other phases as $C_{\mathrm{SR} 1}>C_{\mathrm{SR} 2}$ and $C_{\mathrm{RD} 2}<C_{\mathrm{RD} 3}$.

Let the ratio of the time duration of phase $i$ to the total time duration be denoted by $\tau_{i}\left(\geq 0, \sum_{i} \tau_{i} \leq 1\right)$. The problem of maximization of the end-to-end throughput $t$ can be formulated as the following linear programming problem with optimization variables $\tau_{1}, \tau_{2}$, and $\tau_{3}$.

$$
\begin{aligned}
\operatorname{maximize} & t \\
\text { subject to } & t \leq \tau_{1} C_{\mathrm{SR} 1}+\tau_{2} C_{\mathrm{SR} 2} \\
t & \leq \tau_{2} C_{\mathrm{RD} 2}+\tau_{3} C_{\mathrm{RD} 3} \\
\tau_{1} & \geq 0, \tau_{2} \geq 0, \tau_{3} \geq 0 \\
\tau_{1}+\tau_{2}+\tau_{3} & \leq 1,
\end{aligned}
$$

where the first and the second constraints correspond to the source-to-relay and relay-to-destination links, respectively.

The solutions of problem (??) can be obtained by using linear programming approach and are summarized in Table ??, where $[\cdot]^{\star}$ denotes the optimal solution. We can see that only up to two phases are required, except for the special case, $C_{\mathrm{SR} 2} / C_{\mathrm{SR} 1}+C_{\mathrm{RD} 2} / C_{\mathrm{RD} 3}=1$. This optimal scheduling satisfies the following condition: the total amount of data transmitted from the source is equal to that received at the destination, i.e., $\tau_{1}^{\star} C_{\mathrm{SR} 1}+\tau_{2}^{\star} C_{\mathrm{SR} 2}=\tau_{2}^{\star} C_{\mathrm{RD} 2}+\tau_{3}^{\star} C_{\mathrm{RD} 3}$.

If we do not use phase 2, i.e., if $\tau_{2}=0$, problem (??) will be the maximization problem of end-to-end throughput for HDR. The solutions of this problem are given as

$$
\begin{aligned}
\left(\tau_{1}^{\star}, \tau_{3}^{\star}\right) & =\left(\frac{C_{\mathrm{RD} 3}}{C_{\mathrm{SR} 1}+C_{\mathrm{RD} 3}}, \frac{C_{\mathrm{SR} 1}}{C_{\mathrm{SR} 1}+C_{\mathrm{RD} 3}}\right), \\
t^{\star} & =\frac{C_{\mathrm{SR} 1} C_{\mathrm{RD} 3}}{C_{\mathrm{SR} 1}+C_{\mathrm{RD} 3}} .
\end{aligned}
$$

Hereinafter, this scheduling will be referred to as HDR with transmission scheduling (HDR-TS). Note that the original problem (??) includes HDR-TS; therefore, the end-to-end throughput of the proposed hybrid scheme is greater than or equal to the throughput achieved in HDR-TS.

Theorem 1: Higher end-to-end throughput is achieved in the proposed hybrid scheme than in FDR and HDR-TS when $C_{\mathrm{SR} 2} / C_{\mathrm{SR} 1}+C_{\mathrm{RD} 2} / C_{\mathrm{RD} 3}>1$ and $C_{\mathrm{SR} 2} \neq C_{\mathrm{RD} 2}$.
Proof: We assume $C_{\mathrm{SR} 2} / C_{\mathrm{SR} 1}+C_{\mathrm{RD} 2} / C_{\mathrm{RD} 3}>1$ and $C_{\mathrm{SR} 2}<C_{\mathrm{RD} 2}$. First, we compare the proposed scheme and FDR. The end-to-end throughput of FDR is $\min \left\{C_{\mathrm{SR} 2}, C_{\mathrm{RD} 2}\right\}$. The difference between the end-to-end throughputs of the proposed scheme and FDR is

$$
\begin{gathered}
\frac{C_{\mathrm{SR} 1} C_{\mathrm{RD} 2}}{C_{\mathrm{SR} 1}-C_{\mathrm{SR} 2}+C_{\mathrm{RD} 2}}-\min \left\{C_{\mathrm{SR} 2}, C_{\mathrm{RD} 2}\right\} \\
=\frac{C_{\mathrm{SR} 1} C_{\mathrm{RD} 2}}{C_{\mathrm{SR} 1}-C_{\mathrm{SR} 2}+C_{\mathrm{RD} 2}}-C_{\mathrm{SR} 2}>0 .
\end{gathered}
$$

Then, we compare the proposed scheme and HDR-TS. The difference between the end-to-end throughputs of the proposed scheme and HDR-TS is

$$
\begin{array}{r}
\frac{C_{\mathrm{SR} 1} C_{\mathrm{RD} 2}}{C_{\mathrm{SR} 1}-C_{\mathrm{SR} 2}+C_{\mathrm{RD} 2}}-\frac{C_{\mathrm{SR} 1} C_{\mathrm{RD} 3}}{C_{\mathrm{SR} 1}+C_{\mathrm{RD} 3}} \\
=\frac{C_{\mathrm{SR} 1}^{2} C_{\mathrm{RD} 3}}{\left(C_{\mathrm{SR} 1}-C_{\mathrm{SR} 2}+C_{\mathrm{RD} 2}\right)\left(C_{\mathrm{SR} 1}+C_{\mathrm{RD} 3}\right)} \\
\cdot\left(\frac{C_{\mathrm{SR} 2}}{C_{\mathrm{SR} 1}}+\frac{C_{\mathrm{RD} 2}}{C_{\mathrm{RD} 3}}-1\right)>0 .
\end{array}
$$

The proof for the conditions $C_{\mathrm{SR} 2} / C_{\mathrm{SR} 1}+C_{\mathrm{RD} 2} / C_{\mathrm{RD} 3}>1$ and $C_{\mathrm{SR} 2}>C_{\mathrm{RD} 2}$ is obtained in a similar manner.

In addition, simple switching between FDR and HDR-TS will help achieve the maximum throughput in the case of both FDR and HDR-TS. Therefore, the end-to-end throughput in the proposed hybrid scheme is higher than that in the case of simple switching when $C_{\mathrm{SR} 2} / C_{\mathrm{SR} 1}+C_{\mathrm{RD} 2} / C_{\mathrm{RD} 3}>1$ and $C_{\mathrm{SR} 2} \neq C_{\mathrm{RD} 2}$.

\section{NumeriCAl RESUlTS}

The conventional relaying methods and their end-to-end throughputs are listed below:

- HDR: Setting, $\tau_{1}=\tau_{3}=1 / 2$ and $\tau_{2}=0$. The end-to-end throughput is $t=\min \left\{C_{\mathrm{SR} 1}, C_{\mathrm{RD} 3}\right\} / 2$.

- HDR-TS: Setting, $\left(\tau_{1}^{\star}, \tau_{3}^{\star}\right)$ given in (??) and $\tau_{2}=0$. The expression for the end-to-end throughput is as given in (??).

- FDR: Setting, $\tau_{2}=1$ and $\tau_{1}=\tau_{3}=0$. The end-to-end throughput is $t=\min \left\{C_{\mathrm{SR} 2}, C_{\mathrm{RD} 2}\right\}$.

We show the end-to-end throughput by numerical evaluation by varying $C_{\mathrm{SR} 2}$ and setting $\left(C_{\mathrm{SR} 1}, C_{\mathrm{RD} 2}, C_{\mathrm{RD} 3}\right)=$ (3 Mbps, $2 \mathrm{Mbps}$, $4 \mathrm{Mbps}$ ), as shown in Fig. ??. The behaviors of the relaying methods differ in three parts where (i) $0 \leq C_{\mathrm{SR} 2}<1.5$, (ii) $1.5<C_{\mathrm{SR} 2}<2$, and (iii) $2<C_{\mathrm{SR} 2}$ hold. The first part corresponds to the condition $C_{\mathrm{SR} 2} / C_{\mathrm{SR} 1}+C_{\mathrm{RD} 2} / C_{\mathrm{RD} 3}<1$ given in Table ??, and thus, we get $\tau_{2}^{\star}=0$. Therefore, the proposed scheme in this part is equivalent to the HDR-TS.

The second part, i.e., $1.5<C_{\mathrm{SR} 2}<2$, corresponds to the conditions $C_{\mathrm{SR} 2} / C_{\mathrm{SR} 1}+C_{\mathrm{RD} 2} / C_{\mathrm{RD} 3}>1$ and $C_{\mathrm{SR} 2}<C_{\mathrm{RD} 2}$, and thus, we get $\tau_{3}^{\star}=0$. This is because in phase 2 the amount of data received at the relay is lesser than the amount of data transmitted by the relay; thus, the source should transmit additional data in phase 1 to compensate for this difference. Since the proposed scheme does not involve simple switching between FDR and HDR-TS but switching among three phases, the end-to-end throughput is higher than that in the case of 
TABLE I

OPTIMAL TRANSMISSION SCHEDULING. $x \in[0,1]$.

\begin{tabular}{|c|c|c|c|c|}
\hline \multicolumn{3}{|c|}{ Conditions } & $\left(\tau_{1}^{\star}, \tau_{2}^{\star}, \tau_{3}^{\star}\right)$ & $t^{\star}$ \\
\hline \multicolumn{3}{|c|}{$\frac{C_{\mathrm{SR} 2}}{C_{\mathrm{SR} 1}}+\frac{C_{\mathrm{RD} 2}}{C_{\mathrm{RD} 3}}<1$} & $\tau_{1}^{\star}=\frac{C_{\mathrm{RD} 3}}{C_{\mathrm{SR} 1}+C_{\mathrm{RD} 3}}, \tau_{2}^{\star}=0, \tau_{3}^{\star}=\frac{C_{\mathrm{SR} 1}}{C_{\mathrm{SR} 1}+C_{\mathrm{RD} 3}}$ & $\frac{C_{\mathrm{SR} 1} C_{\mathrm{RD} 3}}{C_{\mathrm{SR} 1}+C_{\mathrm{RD} 3}}$ \\
\hline \multirow{3}{*}{$\frac{C_{\mathrm{SR} 2}}{C_{\mathrm{SR} 1}}+$} & \multirow{3}{*}{$\frac{C_{\mathrm{RD} 2}}{C_{\mathrm{RD} 3}}=1$} & $C_{\mathrm{SR} 2}<C_{\mathrm{RD} 2}$ & $\begin{array}{c}\tau_{1}^{\star}=(1-x) \frac{C_{\mathrm{RD} 3}}{C_{\mathrm{SR} 1}+C_{\mathrm{RD} 3}}+x \frac{C_{\mathrm{RD} 2}-C_{\mathrm{SR} 2}}{C_{\mathrm{SR} 1}-C_{\mathrm{SR} 2}+C_{\mathrm{RD} 2}}, \\
\tau_{2}^{\star}=x \frac{C_{\mathrm{SR} 1}}{C_{\mathrm{SR} 1}-C_{\mathrm{SR} 2}+C_{\mathrm{RD} 2}}, \tau_{3}^{\star}=(1-x) \frac{C_{\mathrm{SR} 1}}{C_{\mathrm{SR} 1}+C_{\mathrm{RD} 3}}\end{array}$ & $\frac{C_{\mathrm{SR} 1} C_{\mathrm{RD} 3}}{C_{\mathrm{SR} 1}+C_{\mathrm{RD} 3}}$ \\
\hline & & $C_{\mathrm{SR} 2}=C_{\mathrm{RD} 2}$ & $\tau_{1}^{\star}=(1-x) \frac{C_{\mathrm{RD} 3}}{C_{\mathrm{SR} 1}+C_{\mathrm{RD} 3}}, \tau_{2}^{\star}=x, \tau_{3}^{\star}=(1-x) \frac{C_{\mathrm{SR} 1}}{C_{\mathrm{SR} 1}+C_{\mathrm{RD} 3}}$ & $\frac{C_{\mathrm{SR} 1} C_{\mathrm{RD} 3}}{C_{\mathrm{SR} 1}+C_{\mathrm{RD} 3}}$ \\
\hline & & $C_{\mathrm{SR} 2}>C_{\mathrm{RD} 2}$ & $\begin{array}{c}\tau_{1}^{\star}=(1-x) \frac{C_{\mathrm{RD} 3}}{C_{\mathrm{SR} 1}+C_{\mathrm{RD} 3}}, \tau_{2}^{\star}=x \frac{C_{\mathrm{RD} 3}}{C_{\mathrm{SR} 2}-C_{\mathrm{RD} 2}+C_{\mathrm{RD} 3}} \\
\tau_{3}^{\star}=(1-x) \frac{C_{\mathrm{SR} 1}}{C_{\mathrm{SR} 1}+C_{\mathrm{RD} 3}}+x \frac{C_{\mathrm{SR} 2}-C_{\mathrm{RD} 2}}{C_{\mathrm{SR} 2}-C_{\mathrm{RD} 2}+C_{\mathrm{RD} 3}}\end{array}$ & $\frac{C_{\mathrm{SR} 1} C_{\mathrm{RD} 3}}{C_{\mathrm{SR} 1}+C_{\mathrm{RD} 3}}$ \\
\hline \multirow{3}{*}{$\frac{C_{\mathrm{SR} 2}}{C_{\mathrm{SR} 1}}+$} & \multirow{3}{*}{$\frac{C_{\mathrm{RD} 2}}{C_{\mathrm{RD} 3}}>1$} & $C_{\mathrm{SR} 2}<C_{\mathrm{RD} 2}$ & $\tau_{1}^{\star}=\frac{C_{\mathrm{RD} 2}-C_{\mathrm{SR} 2}}{C_{\mathrm{SR} 1}-C_{\mathrm{SR} 2}+C_{\mathrm{RD} 2}}, \tau_{2}^{\star}=\frac{C_{\mathrm{SR} 1}}{C_{\mathrm{SR} 1}-C_{\mathrm{SR} 2}+C_{\mathrm{RD} 2}}, \tau_{3}^{\star}=0$ & $\frac{C_{\mathrm{SR} 1} C_{\mathrm{RD} 2}}{C_{\mathrm{SR} 1}-C_{\mathrm{SR} 2}+C_{\mathrm{RD} 2}}$ \\
\hline & & $C_{\mathrm{SR} 2}=C_{\mathrm{RD} 2}$ & $\tau_{1}^{\star}=0, \tau_{2}^{\star}=1, \tau_{3}^{\star}=0$ & $C_{\mathrm{SR} 2}\left(=C_{\mathrm{RD} 2}\right)$ \\
\hline & & $C_{\mathrm{SR} 2}>C_{\mathrm{RD} 2}$ & $\tau_{1}^{\star}=0, \tau_{2}^{\star}=\frac{C_{\mathrm{RD} 3}}{C_{\mathrm{SR} 2}-C_{\mathrm{RD} 2}+C_{\mathrm{RD} 3}}, \tau_{3}^{\star}=\frac{C_{\mathrm{SR} 2}-C_{\mathrm{RD} 2}}{C_{\mathrm{SR} 2}-C_{\mathrm{RD} 2}+C_{\mathrm{RD} 3}}$ & $\frac{C_{\mathrm{SR} 2} C_{\mathrm{RD} 3}}{C_{\mathrm{SR} 2}-C_{\mathrm{RD} 2}+C_{\mathrm{RD} 3}}$ \\
\hline
\end{tabular}

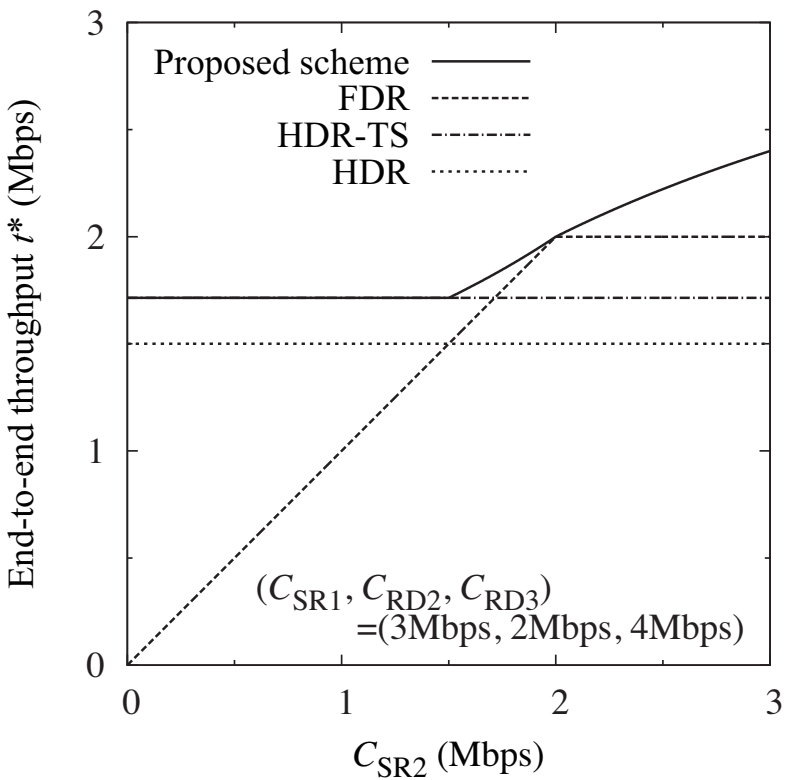

Fig. 2. End-to-end throughput of each relaying schemes.

FDR, HDR-TS, and simple switching between HDR and FDR, as shown in Fig. ??.

Finally, the third part, $2<C_{\mathrm{SR} 2}$, corresponds to the conditions $C_{\mathrm{SR} 2} / C_{\mathrm{SR} 1}+C_{\mathrm{RD} 2} / C_{\mathrm{RD} 3}>1$ and $C_{\mathrm{SR} 2}>C_{\mathrm{RD} 2}$, and thus, we get $\tau_{1}^{\star}=0$. Since the difference in the conditions of the second and the third parts is only the sign of inequality between $C_{\mathrm{SR} 2}$ and $C_{\mathrm{RD} 2}$, the behavior of the third part can be discussed in a manner similar to that in case of the second part. Because switching between FDR and HDR-TS helps achieve the maximum throughput of FDR and HDR-TS, it is apparent that the proposed scheme is superior to the simple switching scheme in the second and the third parts, as shown in Fig. ??

\section{CONCLUSION}

To maximize the end-to-end throughput, three modes for relay can be scheduled in the proposed hybrid scheme: orthogonal reception, orthogonal transmission, and simultaneous reception and transmission at the relay. This maximization problem is solved using linear programming. Numerical evaluations reveal that the throughput of the proposed scheme is higher than that in the case of FDR, HDR, and simple switching between FDR and HDR.

\section{REFERENCES}

[1] H. Suzuki, K. Itoh, Y. Ebine, and M. Sato, "A booster configuration with adaptive reduction of transmitter-receiver antenna coupling for pager systems," Proc. 50th Veh. Tech. Conf. (VTC 1999 Fall), vol.3, pp.15161520 , Sept. 1999.

[2] K. Haneda, E. Kahra, S. Wyne, C. Icheln, and P. Vainikainen, "Measurement of loop-back interference channels for outdoor-to-indoor fullduplex radio relays," Proc. 4th European Conference on Antennas and Propagation (EuCAP 2010), April 2010.

[3] D. Julian, M. Chiang, D. Neill, and S. Boyd, "QoS and fairness constrained convex optimization of resource allocation for wireless cellular and ad hoc networks," Proc. IEEE INFOCOM 2002, June 2002.

[4] R. Cruz and A. Santhanam, "Optimal routing, link scheduling, and power control in multi-hop wireless networks," Proc. IEEE INFOCOM 2003, March-April 2003.

[5] S. Merlin, N. Vaidya, and M. Zorzi, "Resource allocation in multiradio multi-channel multi-hop wireless networks," Proc. IEEE INFOCOM 2008, April 2008.

[6] T. Riihonen, S. Werner, R. Wichman, and E. Zacarias B., "On the feasibility of full-duplex relaying in the presence of loop interference," Proc. 10th IEEE Workshop on Signal Processing Advances in Wireless Communications (SPAWC), June 2009.

[7] T. Riihonen, K. Haneda, S. Werner, and R. Wichman, "SINR analysis of full-duplex OFDM repeaters," Proc. 20th IEEE International Symposium on Personal, Indoor and Mobile Radio Communications (PIMRC), Sept. 2009.

[8] H. Ju, E. Oh, and D. Hong, "Improving efficiency of resource usage in two-hop full duplex relay systems based on resource sharing and interference cancellation," IEEE Trans. Wireless Commun., vol.8, no.8, pp.3933-3938, Aug. 2009.

[9] A. Otyakmaz, R. Schoenen, S. Dreier, and B.H. Walke, "Parallel operation of half- and full-duplex fdd in future multi-hop mobile radio networks," Proc. the 14th European Wireless Conference, pp.1-7, June 2008. 